\title{
Proliferative index and expression of CD38, Zap-70, and CD25 in different lymphoid compartments of chronic lymphocytic leukemia patients
}

Pathology and Laboratory Medicine International

27 January 2011

Number of times this article has been viewed

\author{
Olga Khoudoleeva' \\ Eugeny Gretsov' \\ Natasha Barteneva ${ }^{2,3}$ \\ Ivan Vorobjev' \\ 'Hematology Scientific Center, \\ Russian Academy of Medical Sciences, \\ Moscow, Russia; ${ }^{2}$ Immune Disease \\ Institute and Program in Cellular \\ and Molecular Biology, Children \\ Hospital of Boston, Boston, MA, USA; \\ ${ }^{3}$ Department of Pathology, Harvard \\ Medical School, Boston, MA, USA
}

Correspondence: Ivan Vorobjev Hematology Scientific Center, Russian Academy of Medical Sciences, 4 Novyi Zykovski Proezd,

Moscow 125167, Russia

Tel +7 4956125171

Fax +7 495612 5I 7I

Email ivorobjev@gmail.com

\begin{abstract}
Recent studies of chronic lymphocytic leukemia (CLL) show that malignant B cells proliferate at a rate similar to normal B lymphocytes. This is in apparent contradiction to the very low proliferation rate found in blood specimens from CLL patients. To address this problem, we studied the expression of Ki-67, CD38, CD25, and Zap-70 in different compartments of CLL patients. Using triple-color flow cytometry, we examined the expression of CD38, CD25, Zap-70, and Ki-67 antigens in the peripheral blood, bone marrow, spleen, and lymph nodes biopsies of patients with CLL, splenic marginal zone lymphoma (SMZL), and nonmalignant diseases. In parallel probes of lymph node/spleen biopsies and blood taken from one and the same patient, Ki-67 expression was 17 times higher. Among the whole cohort, we also found significantly higher Ki-67 expression in biopsies from lymph nodes and spleen $(4.95 \% \pm 0.55 \%)$, compared with bone marrow $(1.88 \% \pm 0.32 \%)$ and peripheral blood $(0.45 \% \pm 0.03 \%, P<0.01)$. In CLL patients, there are statistically significant correlations between the expression of CD38 and Ki-67 in bone marrow ( $P \leq 0.01)$, Zap-70 and Ki-67 in blood $(P \leq 0.01)$, and Zap-70 and CD38 in blood $(P \leq 0.01)$. Patients with SMZL also showed a significant correlation between Ki-67 and CD38 expression $(P \leq 0.01)$ and between Ki-67 and Zap-70 expression $(P \leq 0.01)$. We show for the first time that proliferation of B lymphocytes in CLL patients is associated primarily with lymph nodes/spleen. Malignant cells in the blood represent only a subpopulation of nonproliferating and less-activated B cells in this disease.
\end{abstract}

Keywords: chronic lymphoid leukemia, CD38, Zap-70, Ki-67, bone marrow, lymph node

\section{Introduction}

B-cell chronic lymphocytic leukemia (B-CLL) is characterized by large clonal accumulation of $\mathrm{B}$ cells with the abnormal phenotype $\left(\mathrm{CD}^{+} / \mathrm{CD} 23^{+}\right)$and low proliferation capacity. ${ }^{1}$ As the disease is highly heterogeneous, efforts to identify subsets of patients have considered the mutational status of immunoglobulin heavy-chain variable regions $\left(\mathrm{V}_{\mathrm{h}}\right)$, Zap-70 expression, ${ }^{2}$ cytogenetic abnormalities, ${ }^{3} \mathrm{CD} 38$ expression, p53 dysfunction, ${ }^{4}$ as well as other approaches. Among cell-surface markers, both the expression and pathobiological role of CD38 in CLL have been the subject of numerous studies. ${ }^{5-7}$ It has been established that high CD38 expression in B-CLL cells is associated with a worse clinical outcome. ${ }^{8-10}$ Although CD38 is known to participate in signaling transduction in a number of different hematological cell types, ${ }^{11}$ its biological role in CLL tumorigenesis remains unclear. Recent research suggests that a CD38-activated genetic program is relevant in proliferative responses and cell migration. ${ }^{12,13}$ 
A number of biological markers have been recently described that address the prognosis of CLL disease and allow the identification of high-risk patients. However, the majority of these studies were limited by the use of peripheral blood samples. Another important negative prognostic marker in B-CLL is Zap-70, a 70-kDa zeta-chain protein normally essential for T-cell receptor signaling. Zap-70 expression was described in various B-cell malignancies and some subsets of normal B cells. ${ }^{14}$ Flow cytometric evaluation of CD38 in combination with other negative markers such as Zap-70 is an important method for stratifying CLL patients in low-, intermediate-, and high-risk groups and is more straightforward than other technically complex mutational status assays. We analyzed a large cohort of CLL patients undergoing a routine diagnostic study and identified correlations on the primary level in the different lymphoid compartments. The objective of this study was to evaluate the possible correlations between activation markers CD38 and CD25, proliferation index, and Zap-70. We selected Ki-67 as a prominent marker for cell proliferation ${ }^{15}$ and a negative prognostic factor in many types of cancer. ${ }^{16-22} \mathrm{CD} 25$, normally an activation marker on $\mathrm{T}$ cells, was reported to be upregulated on B cells from CLL patients. ${ }^{23-25}$ Analysis of Ki-67 was of particular interest in relation to $\mathrm{CD} 38$, because data on the relationship between CD38 and Ki-67 expression in different B-cell lymphomas remain controversial. ${ }^{26-29}$

Despite the lack of an easily detectable proliferative compartment, recent studies of CLL patients who used oral administration of heavy water $\left({ }^{2} \mathrm{H}_{2} \mathrm{O}\right)$ showed proliferation among B-CLL clonal cells to be substantially more rapid than realized previously with in vivo birth rate of $0.1 \%-1 \%$ of the total leukemic clone per day. ${ }^{30}$ This apparently contradicts the low $\mathrm{Ki}-67$ expression in CLL reported by several authors. ${ }^{27,28}$ To evaluate the possibility that proliferation of B-CLL cells might be restricted to a particular compartment, we performed analysis of activation markers and Ki-67 expression in different biopsy specimens.

\section{Materials and methods}

\section{Patients and sample collection}

Blood samples were collected from 202 consenting patients ( 88 females, 114 males) with CLL. This study included CLL patients seen at the Russian Hematology Scientific Centre between January 2006 and January 2009. Diagnosis of B-CLL was established according to standard morphologic and immunophenotypic criteria. ${ }^{31,32}$ Subject ages ranged from 36 to 80 years (median $=60$ years), and the male-to-female ratio was (1.3:1). The majority of patients $(n=187)$ were untreated at the time of blood collection. Additional bone marrow aspirates $(n=44)$ and biopsies of lymph nodes $(n=32)$ and spleen $(n=9)$ were collected from consented CLL patients. There was no difference in Binet and Rai stages, nor were there significant differences in age or gender between patient groups from which blood samples or bone marrow samples or lymph node sample for biopsy were taken. Patient selection was based on a definitive diagnosis of CLL, and all patients had high- or intermediate-risk CLL. A control group consisting of 32 patients was identified with lymph node and spleen biopsies that were free of any malignant process. The biopsies were processed and stained with a three-color antibody panel in a fashion identical to that described below for CLL patient samples. A second group consisting of 14 patients with splenic marginal zone lymphoma (SMZL) and 7 patients with large B-cell lymphoma were selected from the clinical patients at our institution. Their diagnoses were also established according to standard morphologic and immunophenotypic criteria. ${ }^{31}$

\section{Immunophenotyping for CD38 and CD25 expression}

Fresh samples were taken for surface marker and cytoplasmic marker analysis after obtaining informed consent. Mononuclear cells were obtained from peripheral blood and bone marrow by lysing erythrocytes in ammonium solution via density centrifugation and then kept in phosphate-buffered saline with $0.5 \%$ bovine serum albumin. Mononuclear cells from biopsy material were obtained using Medimachine (BD Biosciences, San Jose, CA, USA) and suspended in a Hanks solution. Surface staining was performed according to manufacturer's protocols using three-color staining. Monoclonal antibodies (direct conjugates) specific for CD19, CD5, CD23, and CD10 were used to define B-CLL phenotype. Antibodies against CD38 and CD25 were used in phycoerythrin (PE) conjugates. Approximately, 500,000 mononuclear cells were stained by adding $10-20 \mu \mathrm{L}$ of conjugated antibodies, followed by incubation for $30 \mathrm{~min}$ at $4{ }^{\circ} \mathrm{C}$. Cells were stained with a mixture of fluorescently labeled antibodies: CD3-FITC/CD19-PE/CD45-PE-Cy5; CD5FITC/CD38-PE/CD19-PE-Cy5; CD10-FITC/CD23-PE/ CD19-PE-Cy5; CD20-FITC/CD25-PE/CD19-PE-Cy5.

Between 30,000 and 50,000 cells were examined for each probe using the FACSCalibur flow cytometer (BD Biosciences). Gating and data analysis were performed 
using CellQuest software (BD Biosciences) and FlowJo software (TreeStar Inc, Ashland, OR, USA). The level of CD38 expression was determined by selective gating as a percentage of $\mathrm{CD} 5^{+} \mathrm{CD} 19^{+}$cells expressing $\mathrm{CD} 38$ (Figure 1). The same method of sample preparation and three-color extracellular staining were used throughout the study period. The level of CD25 expression was determined in the same way using $\mathrm{CD} 20^{+}$instead of $\mathrm{CD} 19^{+}$.

\section{Intracellular flow cytometric analysis of Ki-67 (MIB-I) protein expression}

CLL peripheral blood lymphocytes, bone marrow, and lymph nodes were analyzed by triple-color immunofluorescent staining using antibodies against Ki-67 (Clone MIB-1) and antibodies against CD79a and CD3 (Ki-67-FITC/CD79a-PE/CD3-PECy5). Briefly, purified lymphocytes were fixed in $1 \%$ freshly prepared paraformaldehyde (Sigma, St. Louis, MO, USA) for $5 \mathrm{~min}$ at room temperature. After fixation, the cells were permeabilized using a commercially available kit (BD Biosciences) for $10 \mathrm{~min}$ at room temperature. After staining with Ki-67 (MIB-1)-FITC for $25 \mathrm{~min}$ at $4^{\circ} \mathrm{C}$, cells were washed again and analyzed on a FACSCalibur flow cytometer (BD Biosciences). At least 30,000 events were acquired from each sample. Negative isotype-matched controls (BD Biosciences) were used to exclude nonspecific events. Expression of Ki-67 was determined as a percentage of CD79a-positive cells. Only cases with a negligible percentage of normal B lymphocytes were included in analysis of the cytoplasmic marker.
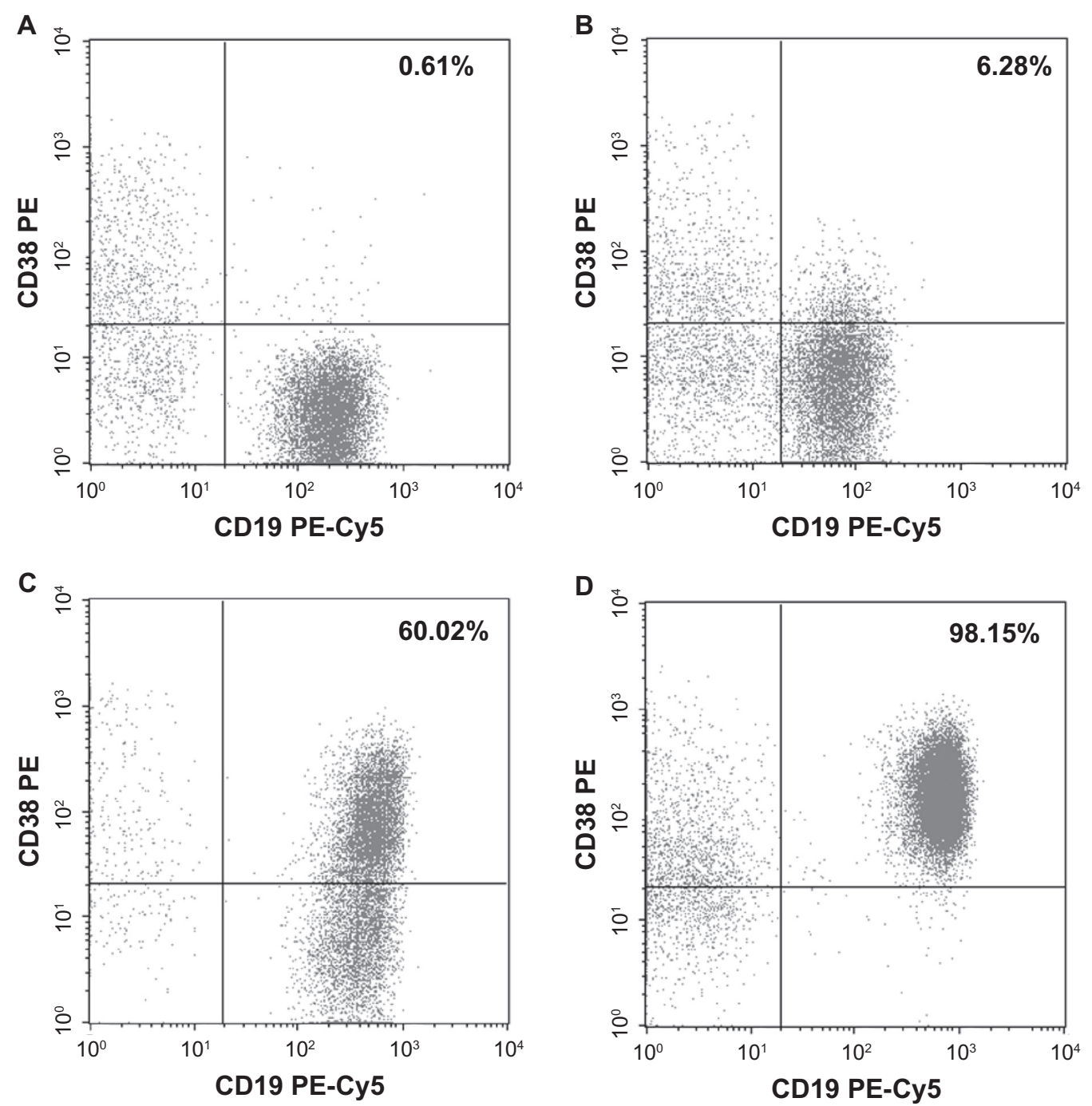

Figure I Representative flow cytometry profiles of CD38 expression in patients with B-CLL. CLL cells were analyzed for surface CD38 expression after incubation with directly conjugated anti-CD 19-PE-Cy5, anti-CD38-PE, and anti-CD5-FITC antibodies. A) and B) Samples from two patients negative for CD38 expression (<30\%). C) Sample of patient positive for CD38 expression (CD38 intermediate, see text for details). D) Sample of patient positive for CD38 expression (CD38 ${ }^{\text {high }}$, see text for details). Numbers are \% of CD38-positive B cells. 


\section{Analysis for Zap-70}

Flow cytometric analysis of intracellular Zap-70 expression was performed on whole blood samples using the method described by Crespo and coauthors. ${ }^{33}$ Briefly, cells were fixed and permeabilized using the BD Cytofix/Cytoperm kit (BD Biosciences) according to the manufacturer's instructions. Antibodies against Zap-70 (Clone 1E7.2) were used in PE conjugate. After staining with Zap-70 antibody, cells were washed again and stained with monoclonal antibody conjugates against $\mathrm{CD} 79 \mathrm{a}$ and $\mathrm{CD} 3$ (BD Biosciences).

To quantify Zap-70 expression, we used the following method of analysis: the identification of the Zap- $70^{+}$cell population was driven by the external isotype-matched control. Analysis of Zap-70 expression in T cells (positive control) was performed by calculating the ratio between the mean fluorescence intensity (MFI) of cells stained with anti-Zap-70 monoclonal antibodies and the MFI of the corresponding isotype-matched control. Biparametric dot plot graphs were obtained for 1) CD3 and Zap-70 or 2) CD79a and Zap-70. Cutoff for Zap-70 expression on B-CLL cells was set at $95 \%$ Zap-70 positivity of T cells (CD79a-FITC/ Zap-70-PE/CD3-PE-Cy5).

\section{Statistical analysis}

The nonparametric Spearman's rank correlation coefficient was applied to evaluate the possible correlation between the continuous variables Zap-70, CD38, CD25, and Ki-67 from blood, lymph nodes, and bone marrow samples from the CLL patient group and the control group with reactive lymph nodes. All computations were carried out using the SSCP statistical program version 17.0 and GraphPad version 5.0 (La Jolla, CA, USA). A value of $P \leq 0.05$ was considered significant for all statistical calculations.

\section{Results \\ CD38 expression}

The level of CD38 expression in CLL in our cohort of patients varied from almost negligible to $100 \%$ (Figure 1). A leukemic cell population was considered positive for CD38 when it was $\geq 30 \%$ in accordance with previous reports. ${ }^{10,34,35}$

We found significantly higher CD38 expression in biopsies from lymph nodes and spleen compared with bone marrow biopsies and peripheral blood (mean percentage, $63.7 \% \pm 5.3 \%$ vs $43.7 \% \pm 5.2 \%$ and $32.0 \% \pm 2.3 \%$ respectively; $P<0.01$ ) (Figure 2). No significant difference was found between lymph node biopsies $(60.7 \% \pm 11.5 \%$; $\mathrm{n}=36)$ and spleen biopsies $(64.4 \% \pm 6.1 \% ; \mathrm{n}=9)$. Thus, we considered them one group for further analysis. Consequently, the percentage of CD38-positive patients identified by probing lymph nodes/spleen, bone marrow, and blood was $68.4 \%, 53.3 \%$, and $38.6 \%$, respectively; $P<0.01$.

The difference in CD38 expression between lymph nodes and blood was confirmed by analysis of parallel specimens (Table 1). In 18 out of 20 cases, the percentage of CD38positive cells in the lymph nodes was higher than in the blood, and in 13 cases, it was at least 1.5 times higher. The overall difference in CD38 expression between the two groups of specimens was highly significant $(P<0.001)$.
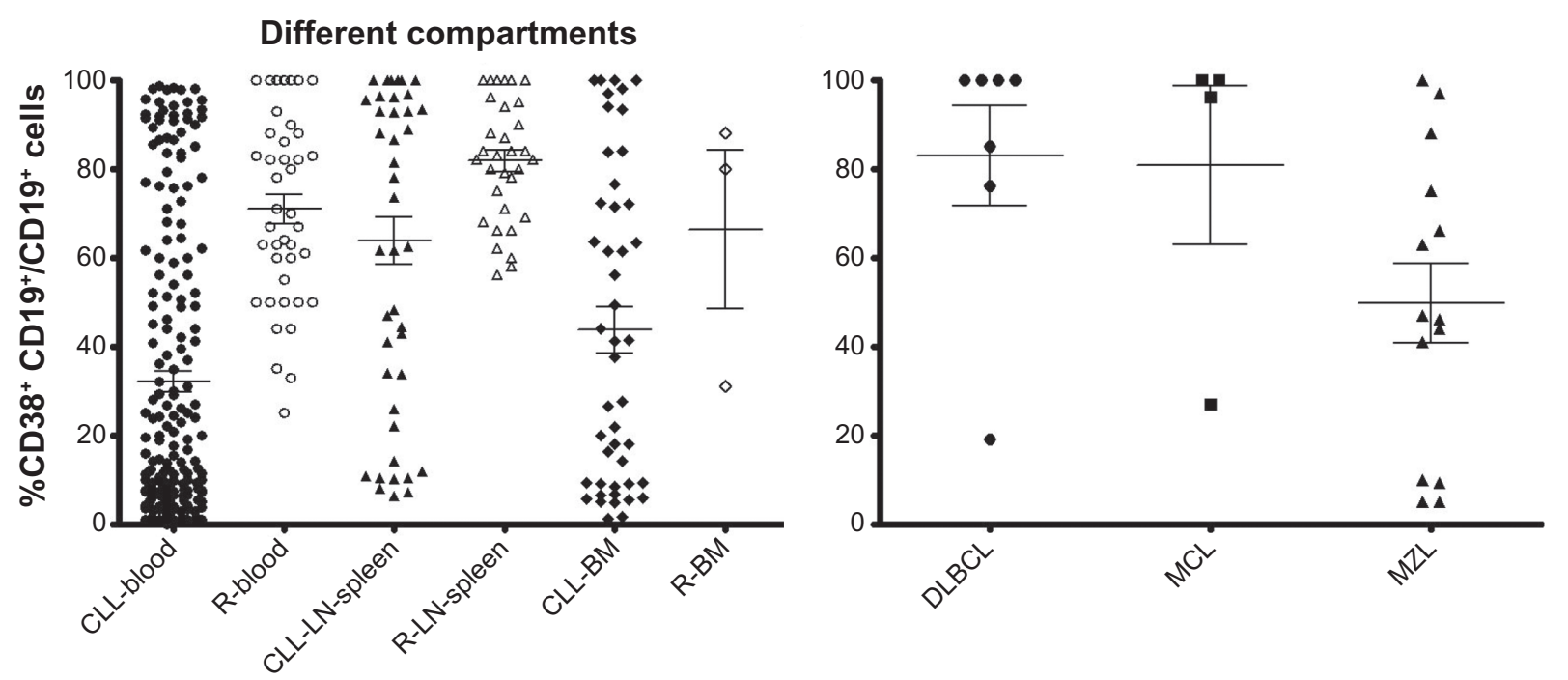

Figure 2 Percentage of $\mathrm{CD}_{3} 8^{+}$cells by lymphoid compartments in patients with B-CLL. Scattergram of CD38 cells in peripheral blood, spleen/lymph nodes, and bone marrow biopsies from CLL patients (the leftmost, center, and rightmost columns of each panel; filled circles, tetrangles, and triangles, respectively). Results are presented as the percentage of $\mathrm{CD} 38^{+}$and $\mathrm{CDI} 9^{+}$, and the means are marked by solid lines.

Abbreviations: CLL, chronic lymphocytic leukemia; LN, lymph node; BM, bone marrow; DLBCL, diffuse large B-cell lymphoma; MZL, marginal zone lymphoma. 


\section{A}

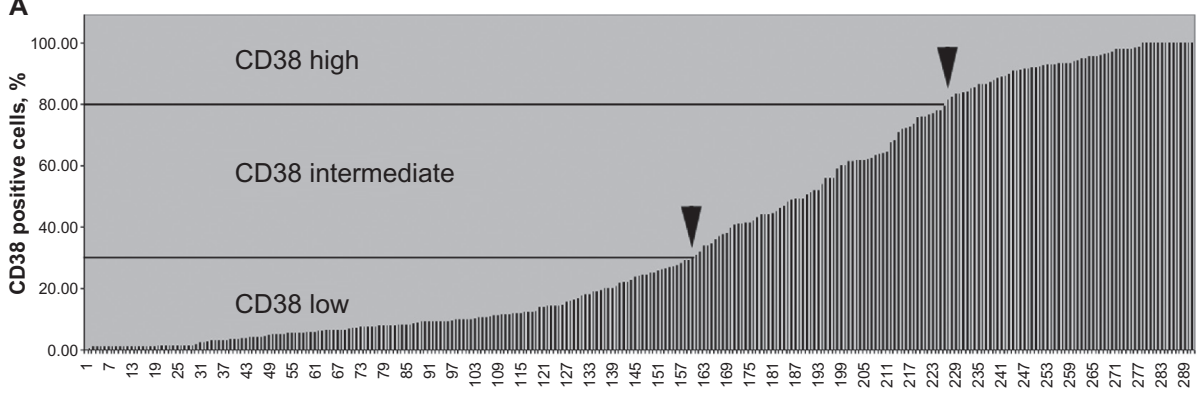

$\mathbf{B}_{100}$
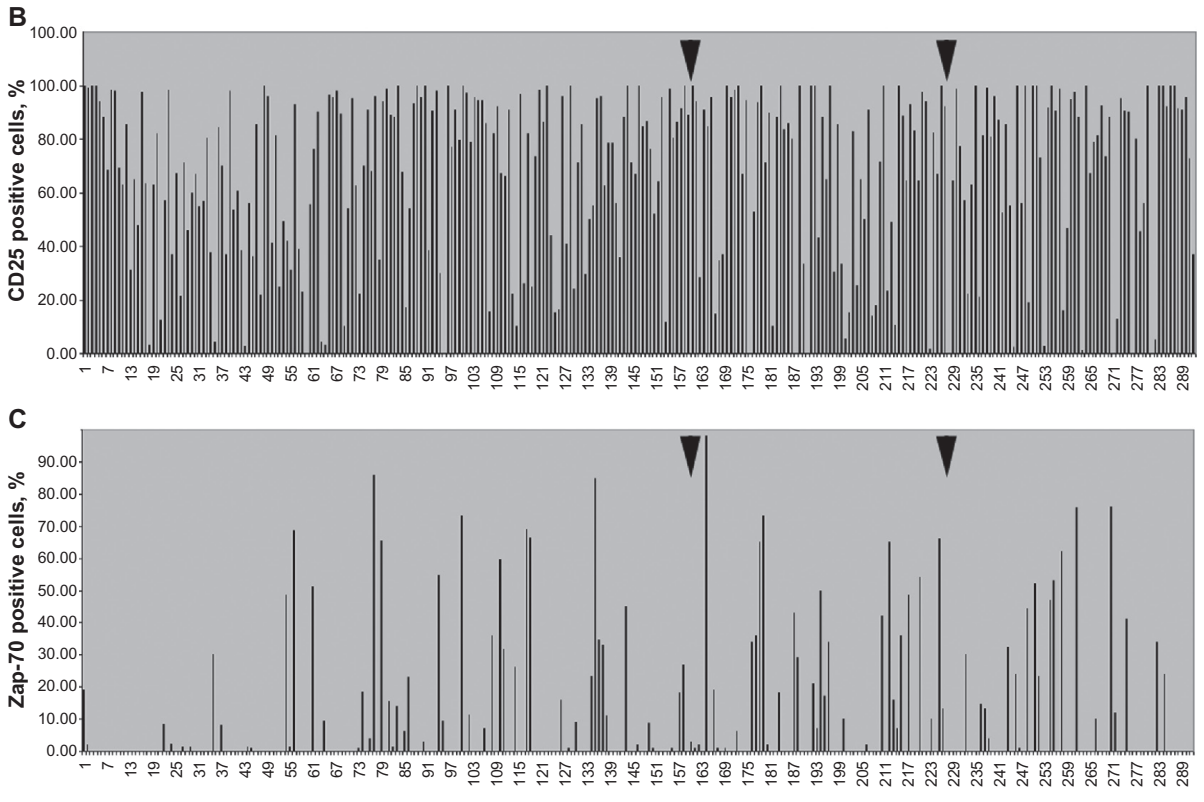

D

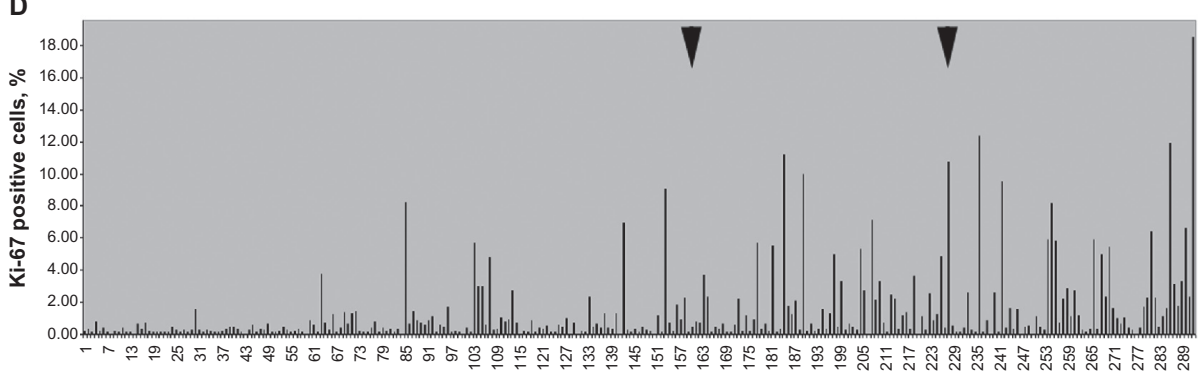

Figure 3 Percentage of different antigens (CD25, Ki-67, and Zap-70) stratified according to CD38 expression. Expression levels of CD25, Zap-70, and Ki-67 from B cells of CLL patients. CLL patients were divided into three groups according to the level of CD38 expression on their B cells: low $(<30 \%)$, intermediate $(30 \%-80 \%)$, and high $(>80 \%)$.

A

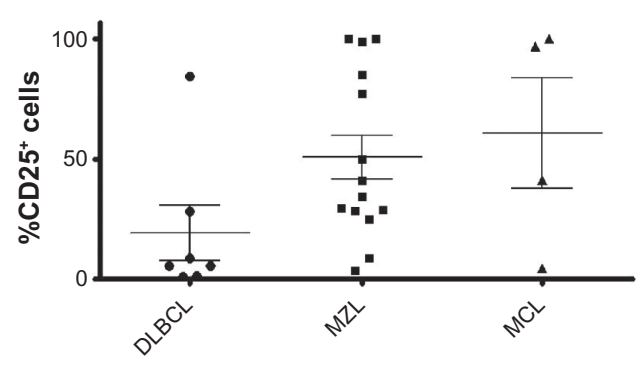

B Different compartments

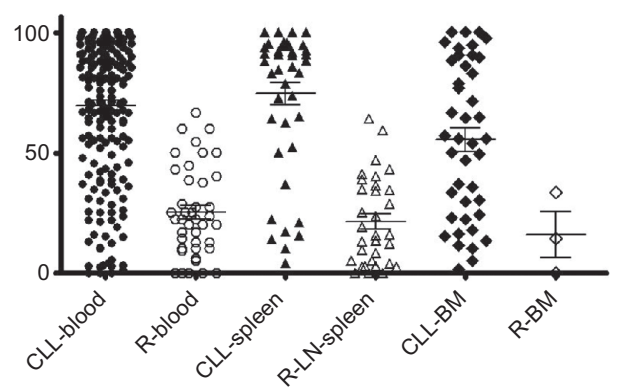

Figure 4 Percentage of $C D 25^{+}$B cells in patients with B-CLL and other lymphomas. Scattergrams of CD25+ B cells in: A) Different B-cell lymphomas and B) different compartments in CLL patients and patients with reactive conditions. Results are presented as the percentage of $C D 25^{+} B$ cells, and the means are marked by solid lines. Abbreviations: CLL, chronic lymphocytic leukemia; LN, lymph node; BM, bone marrow; DLBCL, diffuse large B-cell lymphoma; MZL, marginal zone lymphoma. 
Patients in the SMZL group had slightly higher levels of CD38 expression on B cells $(49.7 \% \pm 8.9 \% ; n=14)$ than CLL patients $(38.6 \% \pm 2.1 \%)$, and patients in the large B-cell lymphoma group had significantly higher levels of CD38 expression $(82.0 \% \pm 11.2 \% ; n=7)$. The level of CD38 expression on $\mathrm{B}$ cells in the reactive specimens was $75.3 \% \pm 2.2 \%$ $(n=76)$. Hence, the level of CD38 expression in B-CLL lymph nodes/spleens was still lower than in reactive lymph nodes/spleens $(63.7 \% \pm 5.3 \%$ vs $81.8 \% \pm 2.4 \% ; P<0.01)$.

The cohort of CLL patients was further stratified according to the level of CD38 expression. Patients were divided into three groups according to the percentage of CD38-positive B-CLL cells (Figure 3): CD $38^{\text {low }}(<30 \%$ of positive cells) (Figure 1A and 1B), CD38 $8^{\text {intermediate }}$ (between $30 \%$ and $80 \%$ of positive cells) (Figure $1 \mathrm{C}$ ), and CD38 ${ }^{\text {high }}$ ( $>80 \%$ of positive cells) (Figure 1D). From the whole cohort $(n=202), 128$ patients had $<30 \%$ of CD38-expressing leukemic cells (CD38 low $), 41$ patients were in the CD38 $8^{\text {intermediate }}$ group, and 33 patients were in the CD $38^{\text {high }}$ group. To further evaluate the relevance of $\mathrm{CD} 38^{\text {high }}$ and $\mathrm{CD} 38^{\text {low }}$, we compared CD38 expression with other markers.

\section{CD25 expression}

Unlike CD38, expression of CD25 on B cells was significantly increased in CLL patients compared with other groups (CLL: $68.4 \% \pm 1.8 \%$; patients with reactive conditions: $23.4 \% \pm 2.2 \%$; SMZL: $50.7 \% \pm 9.2 \%$; and diffuse large B-cell lymphoma (DLBCL): $22.3 \% \pm 13.0 \%$ ) (Figure 4). This finding is in accord with previous reports. ${ }^{24}$ The highest level of CD25 expression in CLL was found in lymph nodes and spleens, and the lowest level was in the bone marrow (Table 2). However, the difference between different compartments was insignificant $(P>0.2)$.

\section{Zap-70 expression}

Using $\mathrm{T}$ cells as an external positive control, we defined the percentage of B cells positive for Zap-70 in different CLL compartments (Figure 5). Expression of Zap-70 in different lymphomas and different compartments in CLL is summarized

Table I Expression of different antigens (\%) on CLL cells in peripheral blood and spleens/lymph nodes from the same patients $(n=20)$

\begin{tabular}{lrr}
\hline & \multicolumn{1}{c}{ CD38 } & \multicolumn{1}{c}{ Ki-67 } \\
\hline Blood & $29.38 \pm 7.03$ & $0.92 \pm 0.30$ \\
Lymph nodes and spleens & $43.84 \pm 7.87$ & $6.80 \pm 1.68$ \\
Ratio' & $2.76 \pm 0.56$ & $17.32 \pm 4.73$ \\
\hline
\end{tabular}

Notes: Mean \pm SEM. 'Ratios were calculated as means for all pairs of specimens.
Table 2 Expression of different antigens (\%) on CLL cells in peripheral blood, bone marrow, and spleens/lymph nodes

\begin{tabular}{llll}
\hline & CD38 & Ki-67 & CD25 \\
\hline Blood $(n=201)$ & $31.98 \pm 2.34$ & $0.45 \pm 0.03$ & $69.46 \pm 2.13$ \\
Bone marrow $(n=42)$ & $44.22 \pm 5.30$ & $1.88 \pm 0.32$ & $55.40 \pm 4.93$ \\
Lymph nodes & $63.7 I \pm 5.32$ & $4.95 \pm 0.55$ & $75.01 \pm 4.63$ \\
$\begin{array}{l}\text { and spleens }(n=42) \\
\text { Reactive lymph }\end{array}$ & $81.78 \pm 2.4 \mathrm{I}$ & $4.76 \pm 0.8 \mathrm{I}$ & $21.25 \pm 3.24$ \\
$\begin{array}{l}\text { nodes and spleens } \\
(\mathrm{n}=32)\end{array}$ & & & \\
\hline
\end{tabular}

Note: Mean \pm SEM.

in Figure 5. Percentages of Zap-70-positive B cells in the peripheral blood of CLL patients ranged from $0 \%$ to $85.4 \%$ $($ median $=13.4)$. The level of Zap-70 expression was significantly higher in the $\mathrm{B}$ lymphocytes of lymph nodes and spleens of B-CLL patients (range: 6\%-98\%; median = 36). Additionally, we assessed Zap-70 expression in samples from patients with SMLZ and large B-cell lymphoma. In SMZL samples, the percentage of Zap-70-positive B cells ranged from $0 \%$ to $28 \%$ (median $=0.5)$ and in DLBCL samples from $0 \%$ to $98 \%$ (median $=31)$. Control samples of peripheral blood from patients (B lymphocytes) without malignancies were negative for Zap-70 staining.

\section{Proliferative index}

The proliferative properties of cells from peripheral blood and solid lymphoid tissues of patients with CLL were estimated
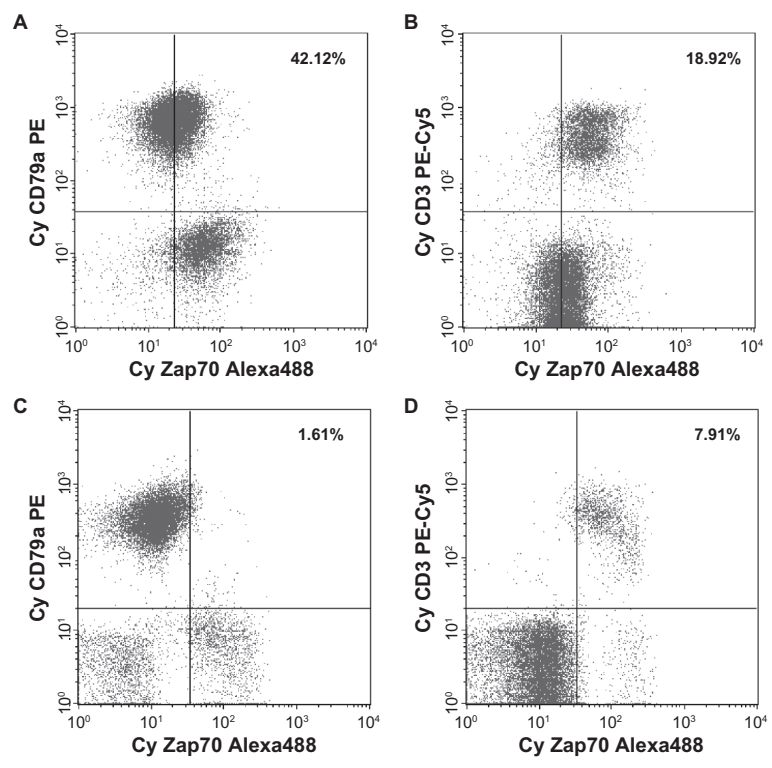

Figure 5 Representative flow cytometry profiles of Zap-70 expression in patients with B-CLL. Peripheral blood cells from B-CLL patients were analyzed by flow cytometry after staining with isotype control or anti-Zap-70 antibodies. A) Dot plots of a Zap-70 negative case. B) Dot plots of a Zap-70-positive case. C) Forward and side scatter of whole blood. D) CD79a and Zap-70-Alexa 488 staining gated on RI lymphocyte scatter gate. 
by determining the expression of the proliferative antigen Ki-67. Some authors have previously reported a very low level of Ki-67 in circulating CLL cells. ${ }^{36}$ Although our results confirm this, we found a rather high percentage of Ki-67-positive B cells in lymph nodes and spleens from CLL patients $(4.94 \% \pm 0.55 \% ; n=45)$ (Figure 6) that was close to Ki-67 levels on B cells in reactive lymph nodes and spleen specimens $(4.76 \pm 0.81 ; \mathrm{n}=32)$. In CLL bone marrow specimens, the percentage was only $1.78 \pm 0.32(\mathrm{n}=44$; $P<0.01)$, and in the peripheral blood, it was 10 times lower, only $0.45 \% \pm 0.03 \%(\mathrm{n}=201 ; P<0.001)$ (Table 1$)$.

Since the difference in Ki-67 expression might be related to variation in patient characteristics (clinical stage, etc), we made direct comparisons of the levels of Ki-67 from parallel peripheral blood and lymph node/ spleen biopsies obtained from 20 patients. The level of proliferation in the lymph nodes/spleens was significantly higher compared to the blood (Table 2; $P<0.005$ ), and this difference was even greater than in independent specimens (Table 1).

Our study indicated that the low level of Ki-67 in peripheral blood may represent a subpopulation of quiescent malignant B cells, while their real proliferation occurs in the lymph nodes and spleen. This view is supported by previous reports of the recovery time for B-CLL cells obtained by nonradioactive labeling. ${ }^{30}$

\section{Correlation analysis of CD38, CD25, Zap-70, and Ki-67 expression in different compartments}

We analyzed possible correlations among CD38, Zap-70, $\mathrm{Ki}-67, \mathrm{CD} 20$, and CD25 expression in peripheral blood, bone

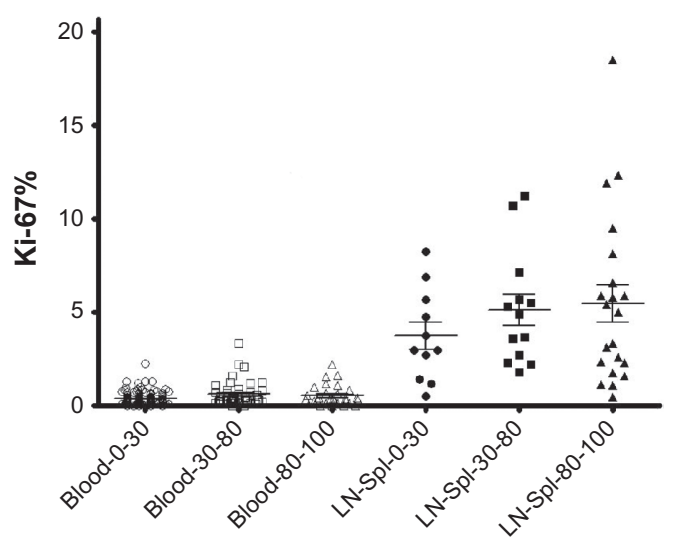

Figure 6 Comparison of $\mathrm{Ki}-67$ versus CD38 in different compartments. Scattergrams of $\mathrm{Ki}-67^{+} \mathrm{B}$ cells in peripheral blood and spleen/lymph node biopsy specimens from CLL patients with CD38 low, intermediate, and high levels. Results are presented as the percentage of $\mathrm{Ki}-67^{+} \mathrm{B}$ cells, and the means are marked by solid lines. Abbreviations: LN, lymph node; Spl, spleen. marrow, and spleens with lymph nodes. A correlation was found among CD38, Zap-70, and Ki-67 in B-CLL (Table 3). However, the correlation between Zap-70 and Ki-67 appeared to be insignificant. In the lymph node and spleen, a positive correlation was found only in the $\mathrm{CD} 38^{\text {intermediate }}$ group.

In patients with SMZL, expression of CD38 correlated with Ki-67 $(r=0.557 ; P<0.01)$ but not with Zap-70 (Table 4).

Levels of CD25 and CD38 expression demonstrated a positive correlation only in lymph nodes and spleens of patients with CLL $(r=0.321 ; P \leq 0.05)$, but there was no significant correlation in blood or bone marrow $(P>0.2)$.

Creating three groups based on CD38 expression levels allowed further evaluation of the relationship among the expression of Ki-67, CD25, and Zap-70. In the overall cohort of patients, Ki-67 was highest in the $\mathrm{CD} 38^{\text {high }}$ group. In blood specimens, Ki-67 was highest in the $\mathrm{CD} 3^{\text {intermediate }}$ group and lowest in the $\mathrm{CD} 38^{\text {low }}$ group. Zap-70 in the whole cohort as well as in blood specimens was only minimal in the CD38 $8^{\text {low }}$ group, slightly higher in $\mathrm{CD} 38^{\text {intermediate }}$, and maximal in the $\mathrm{CD} 38^{\text {high }}$ group. Average expression of $\mathrm{CD} 25$ was nearly the same in all three groups (Table 2).

Our study indicates that in CLL, the levels of expression of CD38 and Ki-67 are significantly higher in lymph nodes/ spleens and bone marrow compared to the peripheral blood.

\section{Discussion}

Most cells circulating in the blood of B-CLL patients are in the G0 phase of the cell cycle, although some of them do express activation markers (CD38 and CD25).

CD38 is a multifunctional surface molecule that is expressed on activated B cells and is involved in signaling transduction and adhesion..$^{5}$ There is a general consensus that $\mathrm{CD} 38$ expression in blood cells is a negative prognostic marker for patients with CLL. ${ }^{8,934,37} \mathrm{CD} 38$ expression in blood cells is considered positively correlated with the majority of negative prognostic markers for CLL, including Zap-70, 38,39 elevated soluble CD23, and cytogenetic abnormalities. ${ }^{35,40,41}$ However, the literature contains no data on comparison of expression of CD38 and proliferative markers in different tissue compartments in CLL.

We compared the levels of CD38 and proliferation markers in different lymphoid compartments of B-CLL patients and control groups of patients, including those with SMZL, large B-cell lymphoma, as well as patients without malignancies. When the percentages of $\mathrm{CD} 38^{+}$cells were plotted for the entire patient population (data not shown), 
Table 3 Expression of different antigens (\%) stratified by CD38 peripheral B-CLL blood, bone marrow, and spleen and lymph nodes

\begin{tabular}{llllll}
\hline & CD38 $_{\text {low }}$ PBL & CD38 $_{\text {intermed- }}$ PBL & CD38 $_{\text {high- }}$ PBL & Bone marrow CLL & Spleen, lymph nodes CLL \\
\hline Zap-70 & $18.4 \pm 2.9(n=68)$ & $29.6 \pm 4.6(n=3 I)$ & $32.1 \pm 5.0(n=2 I)$ & $29.6 \pm 12(n=6)$ & $4 I . I \pm 9.7(n=9)$ \\
Ki-67 & $0.36 \pm 0.34(n=127)$ & $0.66 \pm 0.1(n=42)$ & $0.53 \pm 0.09(n=30)$ & $2.48 \pm 0.77(n=42)$ & $4.95 \pm 0.55(n=42)$ \\
CD25 & $70.0 \pm 2.5(n=127)$ & $69.7 \pm 5.2(n=42)$ & $67.3 \pm 6.1(n=30)$ & $55.4 \pm 4.9(n=42)$ & $75.0 \pm 4.6(n=42)$ \\
\hline
\end{tabular}

Note: Mean \pm SEM.

the patients could be divided into three groups, putting thresholds at $30 \%$ and $80 \%$ of CD38-positive cells. Patients with $30 \%$ or more B cells were considered positive, $30 \%-80 \%$ intermediate positive, $80 \%$ high positive, and those with $<30 \%$ were considered negative.

The expression of CD38 is higher in solid lymphoid organs compared with peripheral blood. ${ }^{42,43}$ We found the expression of CD38 to be different in solid lymphoid organs than in blood, and highest in lymph nodes and spleen biopsies of B-CLL patients. In parallel specimens (lymph node/blood), in 6 out of 20 cases, the difference in the expression of CD38 was large enough to change the grade (from negative to positive and from intermediate to positive high).

Together with CD38, Zap-70 was recently recognized as an independent negative prognostic factor for CLL. ${ }^{14} \mathrm{Zap}-70$, normally expressed in T cells and natural killer cells, ${ }^{44}$ has been reported to be present in human-activated $\mathrm{B}$ cells $\mathrm{s}^{33}$ and also to be expressed by clonal CD19+CD5 ${ }^{+} \mathrm{B}-\mathrm{CLL}$ cells. Recently, it has been shown that Zap-70 phosphorylates after CD38 ligation is a limiting factor for this signaling pathway. ${ }^{45}$ In accordance with previous studies reporting a correlation between CD38 and Zap-70 in peripheral blood, we confirmed a correlation between CD38 and Zap-70 expression in blood and bone marrow, but not in lymph nodes or spleens.

Previous reports have shown that CD25 expression is increased in CLL lymphocytes. ${ }^{23,24}$ Our study revealed a significant correlation between CD25 and CD38 expression only in spleens and lymph nodes of CLL patients and no correlation between CD25 and CD38 in blood. In addition, no correlation was found between CD25 and Ki-67 or Zap-70 among our patients.

Although several studies report that CLL cells from most patients express certain activation-related ${ }^{28,46}$ and cell cycle-related markers, ${ }^{26,47-49}$ data on Ki-67 expression is highly controversial. ${ }^{26,27}$ Our data showing a 10 -fold higher expression of $\mathrm{Ki}-67$ (the mean percentage of expression) in solid tissues (lymph nodes and spleen) of CLL patients are in accordance with the kinetics study of the Messmer Group $^{30}$ and the contention of early CLL researchers ${ }^{50,51}$ that a proliferating component in CLL resides in the solid tissues. Along with histological findings on the presence of proliferation centers in lymph nodes and in the bone marrow, ${ }^{52}$ our data support the hypothesis that the proliferating leukemic cells in CLL always reside in solid lymphoid organs, that is, lymph nodes and spleens. Although our findings show a significant correlation between CD38 and Ki-67 expression in bone marrow, the relationship between CD38 and Ki-67 in lymph nodes/spleens was more complicated. Although the mean percentage of CD38-positive and especially Ki-67-positive populations in this compartment were the highest, we found no significant correlation between them.

In conclusion, our findings give further evidence that activation level of CLL cells does not represent proliferation potential and proliferation of CLL is restricted to the lymph nodes.

Table 4 Spearman's rank order correlations of CD38, Ki-67, CD25, and Zap-70 expression in different lymphoid compartments of CLL patients

\begin{tabular}{llllll}
\hline Correlation pairs & $\begin{array}{l}\text { Lymph nodes } \\
\text { and spleen, CLL }\end{array}$ & Bone marrow, CLL & Blood, CLL & $\begin{array}{l}\text { Lymph nodes and spleen, } \\
\text { nonmalignant }\end{array}$ & $\begin{array}{l}\text { Marginal zone } \\
\text { lymphoma }\end{array}$ \\
\hline CD38/Zap-70 & $0.05 I(n=9)$ & $0.886(n=6)^{*}$ & $0.359(n=106)^{* *}$ & & $-0.044(n=14)$ \\
CD38/Ki-67 & $0.027(n=42)$ & $0.528(n=42)^{* *}$ & $0.246(n=201)^{* *}$ & $-0.295(n=32)$ & $0.557^{* *}(n=14)$ \\
Zap-70/Ki-67 & $0.100(n=9)$ & $0.77 I(n=6)$ & $0.471(n=106)^{* *}$ & & $0.458^{* *}(n=14)$ \\
CD25/CD38 & $0.321(n=42)^{*}$ & $-0.087(n=42)$ & $0.070(n=200)$ & $0.216(n=32)$ & $-0.072(n=14)$ \\
CD25/Ki-67 & $-0.27 I(n=42)$ & $-0.206(n=4 I)$ & $0(n=200)$ & $-0.324(n=32)$ & $-0.103(n=14)$ \\
CD25/Zap-70 & $-0.136(n=9)$ & $-0.100(n=5)$ & $0.084(n=105)$ & & $-0.362 *(n=14)$ \\
\hline
\end{tabular}

Notes: *Significant at $P=0.05 ; * *$ Significant at $P=0.01$. 


\section{Acknowledgments}

This work was partially supported by grants from Russian Foundation for Basic Research \# 08-04-01350 and \# 08-04-01379, and Russian Federation Program grant \# 02.512.11.2296 to Ivan Vorobjev.

\section{Disclosure}

The authors report no conflicts of interest in this work.

\section{References}

1. Chiorazzi N, Rai KR, Ferrarini M. Chronic lymphocytic leukemia. N Engl J Med. 2005;352(8):804-815.

2. Morilla A, Gonzalez de Castro D, Del Giudice I, et al. Combinations of ZAP-70, CD38 and IGHV mutational status as predictors of time to first treatment in CLL. Leuk Lymphoma. 2008;49(11):2108-2115.

3. Döhner H, Stilgenbauer S, Benner A, et al. Genomic aberrations and survival in chronic lymphocytic leukemia. $N$ Engl J Med. 2000; 343(26):1910-1916.

4. Pettitt AR, Sherrington PD, Stewart G, Cawley JC, Taylor AM, Stankovic T. p53 dysfunction in B-cell chronic lymphocytic leukemia: inactivation of ATM as an alternative to TP53 mutation. Blood. 2001; 98(3):814-822.

5. Deaglio S, Vaisitti T, Aydin S, Ferrero E, Malavasi F. In-tandem insight from basic science combined with clinical research: CD38 as both marker and key component of the pathogenetic network underlying chronic lymphocytic leukemia. Blood. 2006;108(4):1135-1144.

6. Bojarska-Junak A, Hus I, Szczepanek EW, Dmoszyńska A, Roliński J. Peripheral blood and bone marrow TNF and TNF receptors in early and advanced stages of B-CLL in correlation with ZAP-70 protein and CD38 antigen. Leuk Res. 2008;32(2):225-233.

7. Patten PE, Buggins AG, Richards J, et al. CD38 expression in chronic lymphocytic leukemia is regulated by the tumor microenvironment. Blood. 2008;111(10):5173-5181.

8. Damle RN, Wasil T, Fais F, et al. Ig V gene mutation status and CD38 expression as novel prognostic indicators in chronic lymphocytic leukemia. Blood. 1999;94(6):1840-1847.

9. Ibrahim S, Keating M, Do KA, et al. CD38 expression as an important prognostic factor in B-cell chronic lymphocytic leukemia. Blood. 2001;98(1):181-186.

10. Eisele L, Haddad T, Sellmann L, Dührsen U, Dürig J. Expression levels of CD38 on leukemic B cells but not on non-leukemic T cells are comparably stable over time and predict the course of disease in patients with chronic lymphocytic leukemia. Leuk Res. 2009;33(6):775-778.

11. Musso T, Deaglio S, Franco L, et al. CD38 expression and functional activities are up-regulated by IFN-gamma on human monocytes and monocytic cell lines. J Leukoc Biol. 2001;69(4):605-612.

12. Deaglio S, Vaisitti T, Aydin S, et al. CD38 and ZAP-70 are functionally linked and mark CLL cells with high migratory potential. Blood. 2007; 110(12):4012-4021.

13. Deaglio S, Aydin S, Grand MM, et al. CD38/CD31 interactions activate genetic pathways leading to proliferation and migration in chronic lymphocytic leukemia cells. Mol Med. 2010;16(3-4):87-91.

14. Wiestner A. Flow cytometry for ZAP-70: new colors for chronic lymphocytic leukemia. Cytometry B Clin Cytom. 2006;70(4):201-203.

15. Gerdes J, Dallenbach F, Lennert K, Lemke H, Stein H. Growth fractions in malignant non-Hodgkin's lymphomas (NHL) as determined in situ with the monoclonal antibody Ki-67. Hematol Oncol. 1984;2(4):365-371.

16. Montebugnoli L, Badiali G, Marchetti C, Cervellati F, Farnedi A, Foschini MP. Prognostic value of Ki67 from clinically and histologically 'normal' distant mucosa in patients surgically treated for oral squamous cell carcinoma: a prospective study. Int J Oral Maxillofac Surg. 2009; 38(11):1165-1172.
17. Jacquemier J, Charafe-Jauffret E, Monville F, et al. Association of GATA3, P53, Ki67 status and vascular peritumoral invasion are strongly prognostic in luminal breast cancer. Breast Cancer Res. 2009; 11(2):R23.

18. Guarneri V, Piacentini F, Ficarra G, et al. A prognostic model based on nodal status and $\mathrm{Ki}-67$ predicts the risk of recurrence and death in breast cancer patients with residual disease after preoperative chemotherapy. Ann Oncol. 2009;20(7):1193-1198.

19. Hasselblom S, Ridell B, Sigurdardottir M, Hansson U, Nilsson-Ehle H, Andersson PO. Low rather than high Ki-67 protein expression is an adverse prognostic factor in diffuse large B-cell lymphoma. Leuk Lymphoma. 2008;49(8):1501-1509.

20. Viale G, Regan MM, Mastropasqua MG, et al. Predictive value of tumor Ki-67 expression in two randomized trials of adjuvant chemoendocrine therapy for node-negative breast cancer. $J$ Natl Cancer Inst. 2008; 100(3):207-212.

21. Neri A, Marrelli D, Pedrazzani C, et al. Prognostic relevance of proliferative activity evaluated by MIB-1 immunostaining in node negative breast cancer. Eur J Surg Oncol. 2008;34(12):1299-1303.

22. Leuenberger M, Frigerio S, Wild PJ, et al. AID protein expression in chronic lymphocytic leukemia/small lymphocytic lymphoma is associated with poor prognosis and complex genetic alterations. Mod Pathol. 2010;23(2):177-186.

23. Hjalmar V, Hast R, Kimby E. Cell surface expression of CD25, CD54, and CD95 on B- and T-cells in chronic lymphocytic leukaemia in relation to trisomy 12 , atypical morphology and clinical course. Eur J Haematol. 2002;68(3):127-134.

24. Sellitto A, de Fanis U, Romano C, et al. Direct or reverse correlations within the expression of activation, differentiation or T-B cooperation molecules on chronic lymphocytic leukemia B cells. Minerva Med. 2003;94(5):331-339.

25. Ding W, Nowakowski GS, Knox TR, et al. Bi-directional activation between mesenchymal stem cells and CLL B-cells: implication for CLL disease progression. Br J Haematol. 2009;147(4):471-483.

26. Jaroslav P, Martina H, Jirí S, et al. Expression of cyclins D1, D2, and D3 and Ki-67 in leukemia. Leuk Lymphoma. 2005;46(11):1605-1612.

27. Bennett F, Rawstron A, Plummer M, et al. B-cell chronic lymphocytic leukaemia cells show specific changes in membrane protein expression during different stages of cell cycle. Br J Haematol. 2007;139(4): 600-604.

28. Damle RN, Temburni S, Calissano C, et al. CD38 expression labels an activated subset within chronic lymphocytic leukemia clones enriched in proliferating B cells. Blood. 2007;110(9):3352-3359.

29. Lin TT, Hewamana S, Ward R, et al. Highly purified CD 38 sub-populations show no evidence of preferential clonal evolution despite having increased proliferative activity when compared with CD38 subpopulations derived from the same chronic lymphocytic leukaemia patient. Br J Haematol. 2008;142(4):595-605.

30. Messmer BT, Messmer D, Allen SL, et al. In vivo measurements document the dynamic cellular kinetics of chronic lymphocytic leukemia B cells. J Clin Invest. 2005;115(3):755-764.

31. World Health Organization Classification of Tumours. Pathology and Genetics of Tumours of Haematopoietic and Lymphoid Tissues. Lyon (France): IARC Press; 2001.

32. Cheson BD, Bennett JM, Grever M, et al. National Cancer Institutesponsored Working Group guidelines for chronic lymphocytic leukemia: revised guidelines for diagnosis and treatment. Blood. 1996;87(12): 4990-4997.

33. Crespo M, Bosch F, Villamor N, et al. ZAP-70 expression as a surrogate for immunoglobulin-variable-region mutations in chronic lymphocytic leukemia. $N$ Engl J Med. 2003;348(18):1764-1775.

34. Hamblin TJ, Orchard JA, Ibbotson RE, et al. CD38 expression and immunoglobulin variable region mutations are independent prognostic variables in chronic lymphocytic leukemia, but CD38 expression may vary during the course of the disease. Blood. 2002;99(3): 1023-1029. 
35. Krober A, Seiler T, Benner A, et al. $\mathrm{V}_{\mathrm{H}}$ mutation status, CD38 expression level, genomic aberrations, and survival in chronic lymphocytic leukemia. Blood. 2002;100(4):1410-1416.

36. Astsaturov IA, Samoilova RS, Iakhnina EI, Pivnik AV, Vorobiov AI. The relevance of cytological studies and Ki-67 reactivity to the clinical course of chronic lymphocytic leukemia. Leuk Lymphoma. 1997; 26(3-4):337-342.

37. Dürig J, Naschar M, Schmücker U, et al. CD38 expression is an important prognostic marker in chronic lymphocytic leukaemia. Leukemia. 2002;16(1):30-35.

38. Dürig J, Nückel H, Cremer M, et al. ZAP-70 expression is a prognostic factor in chronic lymphocytic leukemia. Leukemia. 2003;17(12): 2426-2434.

39. Del Giudice I, Morilla A, Osuji N, et al. Zeta-chain associated protein 70 and CD38 combined predict the time to first treatment in patients with chronic lymphocytic leukemia. Cancer. 2005;104(10):2124-2132.

40. Chevallier P, Penther D, Avet-Loiseau H, et al. CD38 expression and secondary $17 \mathrm{p}$ deletion are important prognostic factors in chronic lymphocytic leukaemia. Br J Haematol. 2002;116(1):142-150.

41. Ottaggio L, Viaggi S, Zunino A, et al. Chromosome aberrations evaluated by comparative genomic hybridization in B-cell chronic lymphocytic leukemia: correlation with CD38 expression. Haematologica. 2003; 88(7):769-777.

42. Ghia P, Guida G, Stella S, et al. The pattern of CD38 expression defines a distinct subset of chronic lymphocytic leukemia (CLL) patients at risk of disease progression. Blood. 2003;101(4):1262-1269.

43. Jaksic O, Paro MMK, Skelin IK, Kusec R, Pejsa V, Jaksic B. CD38 on B-cell chronic lymphocytic leukemia cells has higher expression in lymph nodes than in peripheral blood or bone marrow. Blood. 2004; 103(5):1968-1969.
44. Vivier E, da Silva AJ, Ackerly M, Levine H, Rudd CE, Anderson P. Association of a 70-kDa tyrosine phosphoprotein with the CD16: zeta: gamma complex expressed in human natural killer cells. Eur J Immunol. 1993;23(8):1872-1876.

45. Zubiaur M, Izquierdo M, Terhorst C, Malavasi F, Sancho J. CD38 ligation results in activation of the Raf-1/mitogen-activated protein kinase and the CD3-zeta/zeta-associated protein-70 signaling pathways in Jurkat T lymphocytes. J Immunol. 1997;159(1):193-205.

46. Trentin L, Zambello R, Sancetta R, et al. B lymphocytes from patients with chronic lymphoproliferative disorders are equipped with different costimulatory molecules. Cancer Res. 1997;57(21): 4940-4947.

47. Delmer A, Ajchenbaum-Cymbalista F, Tang R, et al. Overexpression of cyclin D2 in chronic B-cell malignancies. Blood. 1995;85(10): 2870-2876.

48. Wolowiec D, Ciszak L, Kosmaczewska A, et al. Cell cycle regulatory proteins and apoptosis in B-cell chronic lymphocytic leukemia. Haematologica. 2001;86(12):1296-1304

49. Wolowiec D, Wojtowicz M, Ciszak L, et al. High intracellular content of cyclin-dependent kinase inhibitor p27(Kip1) in early- and intermediate stage B-cell chronic lymphocytic leukemia lymphocytes predicts rapid progression of the disease. Eur J Haematol. 2009;82(4): 260-266.

50. Schiffer LM. Kinetics of chronic lymphocytic leukemia. Ser Haematol. 1968;1:3-23.

51. Bazerbashi MB, Reeve J, Chanarin I. Studies in chronic lymphocytic leukaemia. The kinetics of 51Cr-labelled lymphocytes. Scand J Haematol. 1978;20(1):37-51.

52. Caligaris-Cappio F. Role of the microenvironment in chronic lymphocytic leukaemia. Br J Haematol. 2003;123(3):380-388.
Pathology and Laboratory Medicine International

\section{Publish your work in this journal}

Pathology and Laboratory Medicine International is a peer-reviewed, open access journal focusing on innovative basic research and translational research related to pathology or human disease. The journal includes original research, updates, case reports, reviews and commentaries on current controversies. The Academic Sponsor

\section{Dovepress}

of this journal is the Chinese American Pathology Association (CAPA). The manuscript management system is completely online and includes a very quick and fair peer-review system. Visit http://www.dovepress.com/testimonials.php to read real quotes from published authors. 